\title{
Determinação do início da proteção das macieiras 'Fuji' para racionalização do controle químico de Diplocarpon mali
}

\author{
Rosa Maria Valdebenito-Sanhueza ${ }^{1}$; Geraldine de Andrade Meyer'; ${ }^{1}$ Vinícius Adão Bartnicki ${ }^{1}$
}

${ }^{1}$ Proterra Engenharia Agronômica Ltda. BR116, 7320, S2, 95200-000, Vacaria, RS

Autor para correspondência: Rosa Maria Valdebenito Sanhueza (rosamaria@proterra.agr.br)

Data de chegada: 27/02/2014. Aceito para publicação em: 16/06/2014.

$10.1590 / 0100-5405 / 1980$

\section{RESUMO}

Valdebenito-Sanhueza, R.M.; Meyer, G.A.; Bartnicki, V.A. Determinação do início da proteção das macieiras 'Fuji’ para racionalização do controle químico de Diplocarpon mali. Summa Phytopathologica, v.40, n.2, p.182-184, 2014.

O objetivo do trabalho foi avaliar um nível de controle para determinar o início do uso dos fungicidas no manejo da Mancha Foliar de Marssonina (MFM). O método proposto consistiu no monitoramento semanal de 20 macieiras e, quando em quatro plantas foi detectada a presença de sintomas da doença, foi iniciada a aplicação dos fungicidas com intervalos de 10 dias. $\mathrm{Na}$ avaliação do experimento se verificou que todos os tratamentos controlaram a incidência e a severidade da MFM nas folhas. A utilização do nível de controle para MFM reduziu o número de aplicações de fungicidas em $23 \%$ quando comparado com os tratamentos realizados pelo método convencional pelos produtores.

Palavras-chave adicionais: Malus domestica Borkh, Marssonina coronaria, Mancha Foliar de Marssonina, doenças de verão.

\section{ABSTRACT}

Valdebenito-Sanhueza, R.M.; Meyer, G.A.; Bartnicki, V.A. Determination of the beginning of the protection for 'Fuji' apple trees to rationalize the chemical control of Diplocarpon mali. Summa Phytopathologica, v.40, n.2, p.182-184, 2014.

The aim of this study was to assess a control level to determine the beginning of fungicide use in the management of Marssonina Apple Blotch (MAB). The proposed method consisted in the weekly monitoring of 20 apple trees and, when the disease symptoms were detected in four plants, fungicide spraying started at 10-day intervals. All treatments controlled MBA incidence and severity on the leaves. The use of the control level for MBA reduced the number of fungicide sprayings by $23 \%$, compared to the treatments performed by farmers using the conventional method.

Additional keywords: Malus domestica Borkh, Marssonina coronaria, Marssonina apple blotch, summer diseases.

A Mancha Foliar de Marssonina (MFM), causada por Diplocarpon mali Harada \& Sawamura, teleomorfo de Marssonina coronaria Ell. \& J. J. Davis, (sinonímia Marssonina mali Henn.) aumentou sua severidade na década passada no Brasil (2). Citações de epidemias da MFM associadas a períodos de elevada precipitação pluvial e temperaturas de 20 a $22^{\circ} \mathrm{C}$ têm sido relatadas em vários países $(1,3,6)$. Os sintomas foliares da doença são pintas circulares ou irregulares 3 a $10 \mathrm{~mm}$ de diâmetro,freqüentemente com um halo de cor vermelha.

Nas manchas se desenvolvem acérvulos e, nas folhas que caem ao solo no outono, se encontram os apotécios que liberarão ascósporos durante a primavera e verão. Manchas escuras de bordas irregulares se desenvolvem também nos frutos e, no centro delas são encontrados acérvulos (1). Esta doença causa a queda precoce das folhas, reduzindo a área foliar fotossintética e afetando o tamanho, coloração, qualidade e quantidade de frutos, além de enfraquecer o vigor das plantas e a capacidade de produção nos anos seguintes (8).

A eliminação das folhas do pomar, com auxílio de máquinas varredoras, a poda adequada para favorecer a circulação de ar na planta e o controle químico durante o ciclo vegetativo são os métodos de manejo da MFM mais indicados (2). Este último método pode ser realizado com fungicidas do grupo dos ditiocarbamatos, oxicloreto de cobre, benzimidazóis, dodine, ditianona e clorotalonil, aplicados preventivamente ou com programas de fungicidas em intervalos de 25, 15 ou 10 dias $(1,4,7)$. Aplicações a cada 10 dias utilizando tebuconazol, estrobilurinas, ditiocarbamatos e outros fungicidas não penetrantes, além de diminuírem os danos pela MFM, reduzem os prejuízos por podridões de verão $(3,4)$.

O monitoramento das doenças das macieiras é recomendado $(1,7)$, porém ainda não estão definidos os níveis de controle que indiquem o início das pulverizações. Sem essa informação, o uso convencional de defensivos agrícolas causa o aumento no custo de produção e a contaminação dos agroecossistemas. Assim, o objetivo deste trabalho foi comparar tratamentos com fungicidas não penetrantes e penetrantes móveis e um programa com sucessão de ingredientes ativos para manejo da MFM e de outras doenças de verão em macieiras 'Fuji Standard' usando um nível crítico de controle.

Isolados de $M$. coronaria foram obtidos de folhas coletadas em um pomar de macieiras 'Fuji Standard' com sintomas da doença. Para o isolamento do patógeno acérvulos retirados das manchas foram 
esmagados, e suspensos em água destilada esterilizada. Essa suspensão foi distribuída em meio de cultura de batata, dextrose e ágar (BDA), acidificado com ácido láctico. Uma suspensão de $1 \times 10^{5}$ conídios $\mathrm{mL}^{-1}$ de M. mali foi inoculada em três mudas de macieiras 'Fuji Standard' sobre portaenxerto EM9 com seis meses, as quais foram mantidas em câmara úmida em laboratório por sete dias na temperatura de 22 a $25^{\circ} \mathrm{C}$ e, após 21 dias foram determinados os sintomas. Outras três mudas foram mantidas nas mesmas condições como testemunha.

Para o monitoramento e controle químico da MFM no campo, o experimento foi conduzido durante o ciclo 2010/2011 em um pomar de macieiras 'Fuji Standard' sobre portaenxerto EM7, com idade de 12 anos, com histórico de MFM e localizado em Vacaria, RS. O espaçamento foi de 1,2 m entre plantas e 4,0 m entre filas. As coordenadas geográficas da área são $28^{\circ} 25^{\prime} 17^{\prime}$ 'S de latitude e $50^{\circ} 53^{\prime} 38^{\prime}$ 'W de longitude, com altitude de $930 \mathrm{~m}$.

No pomar, uma fileira de plantas foi selecionada. No início dela foram deixadas 10 plantas como bordadura e a partir da $11^{\mathrm{a}}$ foram marcadas 20 plantas de tamanho uniforme. A partir da $3^{\mathrm{a}}$ semana de outubro de 2010 foram monitorados os sintomas de MFM semanalmente. Foi estabelecido que o nível de controle para início dos tratamentos deveria ser quando ocorresse a primeira detecção de sintomas no dossel de quatro plantas das 20 plantas monitoradas.

Os tratamentos usados foram (ingrediente ativo do fungicida, $\mathrm{g}$ ou $\left.\mathrm{mL} 100 \mathrm{~L}^{-1}\right)$ : T1 - trifloxistrobina, 6,00 g (Flint 500WG $\left.{ }^{\circledR}\right)+$ tebuconazol, $12,00 \mathrm{~g}\left(\right.$ Nativo $\left.^{\circledR}\right)$; T2 - propinebe, 210,00 g (Antracol 700WP $\left.{ }^{\circledR}\right)$; T3 - trifloxistrobina, $6,00 \mathrm{~g}+$ tebuconazol, $12,00 \mathrm{~g}+$ propinebe, 210,00 $\mathrm{g}$; T4 - propinebe, 210,00 g + miclobutanil, 4,99 g (Systhane $\left.\mathrm{CE}^{\circledR}\right)$; T5 - ditianona, 56,25 g $\left(\right.$ Delan $\left.^{\circledR}\right)$; T6 - metiram, 210,00 g (Poliram $\left.\mathrm{DF}^{\circledR}\right)$; $\mathrm{T} 7$ - Programa: Uma sucessão de fungicidas: ditianona, 56,25 g; propinebe, $210,00 \mathrm{~g}$; miclobutanil, 4,99 g; propinebe, 210,00 g; fluazinam, 50,00 $\mathrm{g}\left(\right.$ Frowncide 500SC $\left.{ }^{\circledR}\right)$; mancozebe, $240,00 \mathrm{~g}$ (Dithane $\mathrm{NT}^{\mathbb{B}}$ ), trifloxistrobina 5,00 g; trifloxistrobina, 6,00 g + tebuconazole, $12,00 \mathrm{~g}$; difenoconazol, 3,50 mL + ditianona, 56,25 g; T8 - mancozebe, 240,00 g; e T9 - testemunha (sem tratamento).

As aplicações foram realizadas com pulverizador costal Jacto ${ }^{\circledR}$ de $20 \mathrm{~L}$, com bico cônico e a calda foi pulverizada até o ponto de escorrimento e aplicadas em intervalos de 10 dias até 27/03/2011. Para a avaliação da MFM, em 06/04/2011 foram coletadas 50 folhas por parcela e determinada a incidência (percentagem de folhas doentes) e a severidade (número de lesões por folha). Na mesma data, que coincidiu com a maturação comercial dos frutos, foram coletadas 80 maçãs por parcela e, após a hidratação delas e incubação a $22^{\circ} \mathrm{C}$ por 30 dias na temperatura de 15 a $18^{\circ} \mathrm{C}$ foram registrados os sintomas das podridões de verão. Quatro plantas da área tratadas pelo produtor foram avaliadas quanto à presença de sintomas da doença nas folhas.

O delineamento foi em blocos casualizados com quatro repetições e cada parcela constituída de três plantas, sendo utilizada a planta central como área útil. Para análise da variância dos dados utilizou-se o programa Assistat (5) e as médias foram comparadas com o teste de Scott-Knott $(\mathrm{p}<0,05)$.

Nas mudas de macieiras 'Fuji Standard', aos 21 dias após a inoculação de $D$. mali foram observados os sintomas da doença em todas as folhas.

No pomar, na última semana de novembro (23/11/2010), o nível de controle foi atingido, uma vez que se detectou a doença em quatro plantas marcadas. Assim, em 27/11/2010 iniciaram-se os tratamentos que foram realizados independentemente da precipitação acumulada durante os intervalos. Em um longo período de chuva que se estendeu do início da segunda quinzena de janeiro até a o final da primeira quinzena de fevereiro, ocorreu o acúmulo de $304 \mathrm{~mm}$ de precipitação (Figura 1).

Apesar da alta quantidade de chuva, não se detectou redução no controle entre os tratamentos (Tabela 1). A doença progrediu rapidamente nas plantas sem tratamento (testemunha), sendo que no término do experimento apresentavam desfolha intensa. Durante o experimento foram realizadas 13 aplicações de fungicidas, enquanto o produtor realizou 17 aplicações, ou seja, quatro aplicações adicionais para o manejo da doença. $\mathrm{Na}$ área do produtor, se observou 3,0\% e $3,3 \%$ de incidência e severidade, respectivamente, na mesma data de avaliação do experimento.

Não se observou fitotoxicidade em nenhum dos tratamentos realizados nas macieiras. Em algumas maçãs armazenadas das testemunhas se observaram sintomas da MFM.

Todos os tratamentos reduziram igualmente a incidência e a severidade da MFM em percentagens que variaram em $83 \%$ a $92 \%$ e $90 \%$ a $98 \%$, respectivamente (Tabela 1). Dentre as doenças de verão, a podridão olho-de-boi (Cryptosporiopsis perennans) predominou na área

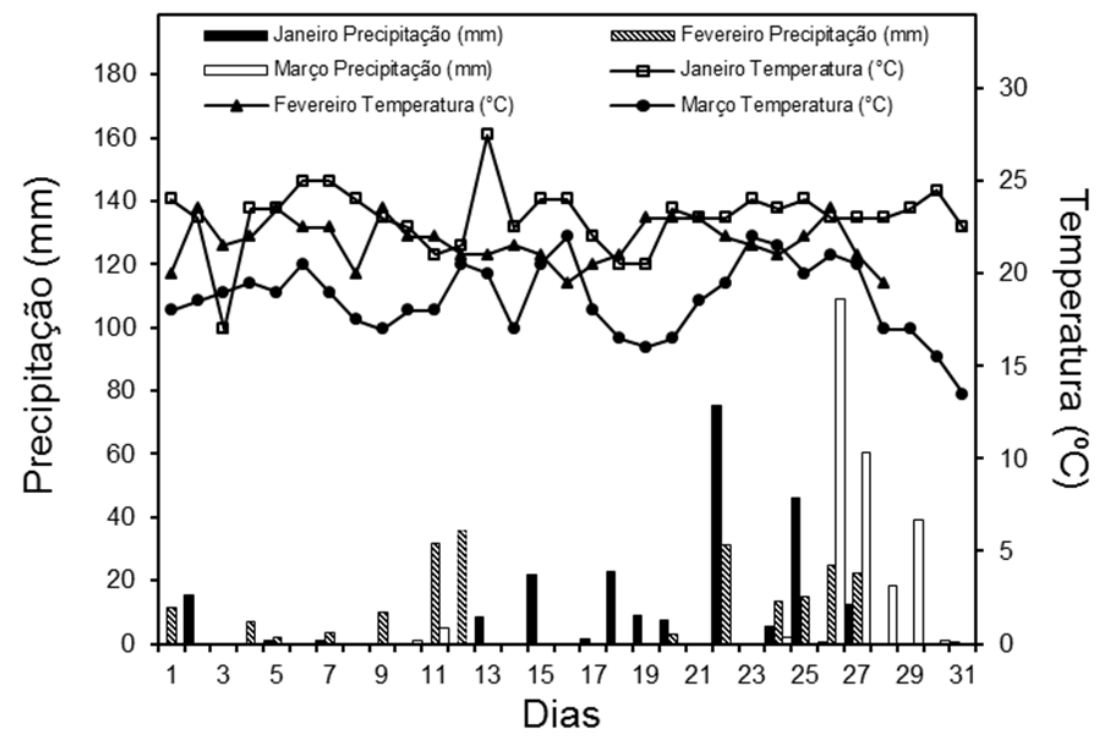

Figura 1. Temperatura média diária e precipitação pluvial nos meses de janeiro a março de 2011. 
Tabela 1. Avaliação de fungicidas pulverizados a partir do nível crítico de incidência e com intervalos de 10 dias para o controle da Mancha Foliar de Marssonina (MFM) e das podridões de verão das maçãs na cv. Fuji Standard em Vacaria, RS (Ciclo 2010-2011).

\begin{tabular}{|c|c|c|c|c|c|}
\hline Tratamentos/ (g ou mL de i.a. 100L $\left.\mathrm{L}^{-1}\right)$ & $\begin{array}{c}\text { Controle (\%) } \\
\text { da incidência da } \\
\text { MFM }^{1}\end{array}$ & $\begin{array}{c}\text { Controle (\%) da } \\
\text { severidade da } \\
\text { MFM }\end{array}$ & $\begin{array}{c}\text { Incidência } \\
\text { (\%) da POB } \\
\text { nos frutos }^{2}\end{array}$ & $\begin{array}{l}\text { Incidência } \\
\text { (\%) da PA } \\
\text { nos frutos }\end{array}$ & $\begin{array}{c}\text { Incidência (\%) } \\
\text { da PB nos } \\
\text { frutos }\end{array}$ \\
\hline 1. Trifloxistrobina, $6 \mathrm{~g}+$ Tebuconazol, $12 \mathrm{~g}$ & $85,88^{\text {ns }}$ & 94,59 ns & $4,10 \mathrm{~b}$ & $1,25^{\mathrm{ns}}$ & $0,25^{\text {ns }}$ \\
\hline 2. Propinebe, $210 \mathrm{~g}$ & 92,06 & 98,45 & 8,10 a & 0,50 & 0,00 \\
\hline 3. Trifloxistrobina, $6 \mathrm{~g}+$ Tebuconazol, $12 \mathrm{~g}+$ Propinebe, $210 \mathrm{~g}$ & 84,12 & 90,73 & $7,20 \mathrm{a}$ & 0,25 & 0,50 \\
\hline 4. Propinebe, $210 \mathrm{~g}+$ Miclobutanil, 4,99g & 83,24 & 94,28 & $13,40 \mathrm{a}$ & 0,00 & 0,00 \\
\hline 5. Ditianona, $56,25 \mathrm{~g}$ & 89,41 & 96,68 & $8,10 \mathrm{a}$ & 0,75 & 0,75 \\
\hline 6. Metiram, $210 \mathrm{~g}$ & 88,53 & 96,99 & $3,10 \mathrm{~b}$ & 0,50 & 0,00 \\
\hline 7. Programa ${ }^{3}$ & 92,94 & 95,29 & $0,60 \mathrm{~b}$ & 0,00 & 1,00 \\
\hline 8. Mancozebe, $240 \mathrm{~g}$ & 86,76 & 94,74 & $6,60 \mathrm{a}$ & 0,50 & 0,00 \\
\hline 9. Testemunha & $(28,25)$ & $(11,51)$ & $9,10 \mathrm{a}$ & 0,25 & 0,50 \\
\hline CV (\%) & 11,91 & 9,45 & 35,33 & - & - \\
\hline
\end{tabular}

${ }^{1}$ Médias de quatro blocos por tratamento, sendo cada parcela composta por três plantas, sendo útil a central, de onde foram retiradas 50 folhas para registro da MFM, 80 maçãs para avaliação das podridões. ${ }^{2}$ POB: Podridão olho-de-boi; PA: podridão amarga; PB: podridão branca

${ }^{3}$ Uma sucessão de fungicidas: ditianona, 56,25 g de i.a; propinebe, 210,00 g; miclobutanil, 4,99 g; propinebe, 210,00 g; fluazinam, 50,00 g; mancozebe, $240 \mathrm{~g}$; trifloxistrobina, 5,00 g; trifloxistrobina, $6,00 \mathrm{~g}+$ tebuconazol, $12,00 \mathrm{~g}$; difenoconazol, 3,50 $\mathrm{mL}+$ ditianona, 56,25 g.

experimental. O fungicida metiram (T6), trifloxistrobina + tebuconazol (T1) e o programa avaliado (T7) reduziram a incidência da podridão olho-de-boi em $56 \%$, 93\% e $66 \%$, respectivamente.

Os fungicidas recomendados na literatura para a MFM, associados ou em sucessão, foram igualmente eficientes quando avaliados nas condições de Vacaria, RS. Neles se incluem os ditiocarbamatos, as estrobilurinas e a ditianona, e em dose menor que a usada na Coréia do Sul (3). O nível de controle usado na macieira para MFM que avalia todo o dossel das plantas diferiu do usado para entomosporiose, ainda não validado experimentalmente, no que se recomenda coletar 20 folhas dos ramos do terço inferior da planta (9). A estratégia de controle químico através de um nível crítico de controle reduziu a intensidade de MFM e foi eficiente no controle dos patógenos associados às doenças de verão. Assim, o método é promissor para racionalizar o uso de fungicidas e reduzir os danos causados pela MFM e pelas podridões de verão em 'Fuji Standard'.

\section{REFERÊNCIAS}

1. Harada, Y.; Sawamura, K.; Konno, K. Diplocarpon mali, sp.nov. the perfect stage of apple blotch fungus Marssonina coronaria. Japanese Journal of Phytopathology, Tokyo, v.40, n.5, p.412-428, 1974.

2. Kretzschmar, A.A., Marodin, G.A.B., Duarte, V. Ocorrência e intensidade de
Marssonina mali em macieiras cv. Eva nas condições da Depressão Central do Rio Grande do Sul. Revista de Ciências Agroveterinárias, Lages, v.4, n.2, p. 145-147, 2005.

3. Lee, D.H.; Shin, H.C.; Cho, R.H.; Uhm, J.Y. Development of a 15-day Interval Spraying Program for Controlling Major Apple Diseases. Plant Pathology Journal, New York, v.24, n.4, p.439-446, 2008.

4. Lee, D.H.; Shin, H.C.; Cho, R.H.; Uhm, J.Y. Reducing Fungicidal Spray Frequency for Major Apple Diseases by Increasing the Spray Interval from 15 to 25 days. Plant Pathology Journal, New York, v.25, n.3, p.270-279, 2009.

5. Silva, F. de A.S.E.; Azevedo, C.A.V. Principal Components Analysis in the Software Assistat-Statistical Attendance. In: World Congress on Computers in Agriculture, 4, Orlando- Florida, EUA: Anais...American Society of Agricultural and Biological Engineers, p. 393-396, 2006.

6. Tamietti, G.; Matta, A. First Report of Leaf Blotch Caused by Marssonina coronaria on Apple in Italy. Plant Disease, Saint Paul (Minnesota), v.87, n.8, p.1005, 2003.

7. Valdebenito-Sanhueza, R.M.; Spolti, P.; Del Ponte, E. M. Controle do inóculo inicial para redução dos danos da podridão olho de boi em macieiras. Revista Brasileira de Fruticultura, Jaboticabal, v.32, n.4, p. 1044-1054, 2010.

8. Yin, L.; Zou, Y.; Li, M.; Ke, X.; Li, C.; Liang, D.; Ma, F. Resistance of Malus plants to Diclarpon mali infection is associated with the antioxidant system and defense signaling pathways. Physiological and Molecular Plant Pathology, Michigan, v.64, p.146-152, 2013.

9. Zwett, Van Der T.; Biggs, A.R. Fabraea Leaf spot, Fabraea maculata. Extension Service West Virginia University, Morgantown. Disponível em: $<$ http://www.caf.wvu.edu/kearneysville/disease descriptions/omfabrea $>$ omfabrea.html. Acesso em 10 de fev. 2014. 